\title{
Health Literacy and Physical Activity: A Systematic Review
}

\author{
Alessandra Buja, Andrea Rabensteiner, Milena Sperotto, Giulia Grotto, Chiara Bertoncello, \\ Silvia Cocchio, Tatjana Baldovin, Paolo Contu, Chiara Lorini, and Vincenzo Baldo
}

\begin{abstract}
Background: The importance of health literacy (HL) in health promotion is increasingly clear and acknowledged globally, especially when addressing noncommunicable diseases. This paper aimed to collect and summarize all current data from observational studies generating evidence of the association between HL and physical activity (PA) and to analyze intervention studies on the promotion of PA to ascertain whether HL moderates the efficacy of such intervention. Methods: A comprehensive systematic literature search of observational studies investigating the association between HL and PA was performed. Intervention studies on the promotion of PA that also measured the HL levels of participants and its effect on the outcome of the intervention were also identified. Results: Of the 22 studies included in this review, 18 found a significant positive association between high HL and high levels of PA. The only intervention study among them indicated that HL was not a significant moderator of the intervention's effectiveness. Conclusion: HL can enable individuals to make deliberate choices about their PA and thus contribute to preventing many chronic noncommunicable diseases. That said, low levels of HL do not seem to influence the efficacy of health promotion interventions.
\end{abstract}

Keywords: exercise, health behavior, health promotion, prevention

Worldwide, efforts to promote health and prevent disease have become more necessary than ever in order to address rising levels of noncommunicable diseases and related disabilities. ${ }^{1}$ Health literacy (HL) seems to play a part in health promotion interventions, and its influence on actions to promote health was stressed at the Ninth Global Conference on Health Promotion., ${ }^{2,3}$ Kickbusch and $\mathrm{Maag}^{4}$ defined HL as the ability of individuals to make decisions in their everyday lives that have a positive impact on their health. It enhances individuals' self-determination and healthrelated behavior, improving their ability to find and understand health information, and enabling them to take responsibility for their health. ${ }^{4}$ Conversely, a limited HL has an adverse impact on health-related behavior and disease prevention strategies, such as participation in screening programs. ${ }^{5-9}$ In addition, and partly as a consequence of their often adopting an unhealthy behavior, individuals with a low HL tend to make more use of health care services, including hospitalization and emergency services. ${ }^{10-12}$

A well-known factor that promotes a good state of health and helps to prevent noncommunicable diseases is physical activity (PA). ${ }^{13}$ One of the leading modifiable risk factors for global mortality is physical inactivity. Sedentary individuals have an estimated $20 \%$ to $30 \%$ higher risk of premature death than individuals who are physically active. ${ }^{14} \mathrm{~A}$ study published in 2012 found that physical inactivity accounted for 9\% (range 5.1\%-12.5\%) of premature mortality worldwide. ${ }^{15}$ It was independently responsible for a proportion of the risk of several chronic diseases, including $6 \%$ (range 3.2\%-8\%) of coronary heart disease, 7\% (range 3.9\%-9.6\%) of type II diabetes mellitus, and $10 \%$ (range $5.6 \%-14.1 \%$ ) of breast cancer, as well as considerably raising the risk of colon cancer (range

Buja, Rabensteiner, Sperotto, Grotto, Bertoncello, Cocchio, Baldovin, and Baldo are with the Department of Cardiological, Thoracic, Vascular Sciences and Public Health, University of Padova, Padova, Italy. Lorini is with the Department of Health Sciences, University of Florence, Firenze, Italy. Contu is the Department of Medical Sciences and Public Health, University of Cagliari, Monserrato, Cagliari, Italy. Buja (alessandra.buja@unipd.it) is corresponding author.
5.7\%-13.8\%). ${ }^{14,16} \mathrm{PA}$, on the other hand, is also associated with an enhanced cognitive function and mental health across the life span, as well as a better overall health and physical functioning. ${ }^{16}$ To our knowledge, there have so far been no literature reviews on the association between PA and HL capable of shedding light on this claimed contribution of HL to health promotion.

The aim of this systematic review was to collect and summarize all currently available evidence emerging from observational studies on the association between HL and an active lifestyle to see if empirical data confirm the conviction that HL can influence people's PA levels. In addition, intervention studies promoting PA and assessing the $\mathrm{HL}$ of participants were also considered to ascertain whether HL is a moderator of an intervention's effectiveness.

\section{Methods}

\section{Search Strategy and Data Sources}

For the present study, a comprehensive and systematic literature search was conducted in the MEDLINE and Scopus databases to identify:

1. Observational studies (cross-sectional, cohort, and case-control studies) investigating the association between $\mathrm{HL}$ and PA, and

2. Intervention studies aiming to promote PA, which examined whether HL acted as a moderator of the efficacy of the intervention.

The search process involved combining the terms "health literacy" or "literacy" with the terms "physical activ*," "sedentary," "sedentarity," "gym*," "physical inactiv*," and "physical exerc*" using Boolean operators. The authors also checked the reference lists of the papers included in the review for any articles not already considered.

\section{Eligibility Criteria}

The studies included in the review had to meet the following inclusion criteria: HL had to be measured using validated 
questionnaires, a measure of the association between HL and PA had to be reported, the paper had to have been published from January 2000 to March 2018, and written in English. Studies involving patients with a specific disease were excluded.

The search strings were defined as reported in Supplementary Material 1 (available online).

\section{Study Selection and Data Extraction}

The initial search yielded 239 articles in PubMed and 235 in Scopus. After the search was completed, the records retrieved were imported to Endnote and duplicates were removed. Two reviewers (M.S. and G.G.) checked the search hits by reading the article titles and abstracts. If the results of a study were published more than once, only the most complete article was considered in the analysis.

The material was qualitatively assessed by collecting the following data from each study: first author's name, year of publication, journal, study design, sampling method, characteristics of the study sample (eg, age range), measures of outcome and exposure, results, confounding factors, interactions, and the authors' conclusions. The results are presented by age group.

\section{Quality Assessment Criteria}

Two different authors (M.S. and G.G.) independently judged the methodological quality of the studies using the Strengthening the Reporting of Observational Studies in Epidemiology (STROBE) approach $^{17}$ for observational studies and the Consolidated Standards of Reporting Trials (CONSORT) approach ${ }^{18}$ for intervention studies.

The STROBE Statement is a checklist of 22 items that relate to the article's title and abstract (item 1), the introduction (items 2 and 3 ), methods (items 4-12), results (items 13-17), discussion sections (items 18-21), and other information (item 22 on funding). ${ }^{17}$ Eighteen items are common to all 3 designs (cohort, case-control, and cross-sectional studies), whereas 4 (items 6,12,14, and 15) are design-specific, with different versions for all or part of the item. ${ }^{17}$ On the other hand, the CONSORT checklist is a 25-item instrument that focus on reporting how randomized trials was designed, analyzed, and interpreted. ${ }^{18}$ The items relate to the article's title and abstract (items $1 \mathrm{a}$ and $1 \mathrm{~b}$ ), the introduction (items $2 \mathrm{a}$ and $2 \mathrm{~b}$ ), methods (items 3a-12b), results (items 13a-19), discussion sections (items 20-22), and other information (items 23-25). ${ }^{18}$ Total STROBE and CONSORT scores were calculated for each study by 2 different authors independently. A higher percentage of items conforming to the guidelines indicated a higher methodological quality. Cohen kappa was calculated to establish the level of agreement between the 2 reviewers' assessments. ${ }^{19}$

\section{Results}

After the authors had reviewed all the abstracts, 74 studies were judged potentially relevant for the purposes of this review, and the full texts were obtained and assessed independently by the reviewers.

After assessing the 74 full texts for eligibility, 18 of the articles met our exclusion and inclusion criteria. The other articles were rejected for the following reasons: 38 included no HL measurement, 13 did not measure PA, and 5 did not provide a measure of the association between HL and PA. The check on the reference lists of the 18 eligible articles yielded another 4 papers that matched our inclusion and exclusion criteria.
Two different authors (M.S. and G.G.) independently judged the methodological quality of the included studies using the STROBE approach ${ }^{17}$ for observational studies and the CONSORT approach $^{18}$ for intervention studies. A Cohen kappa of .926 reflected a very good agreement between the 2 reviewers.

Figure 1 shows a flow diagram of the article selection process.

The study selection process led to the identification of a total of 22 studies that met the inclusion criteria of this review. The majority of the studies had adopted a cross-sectional design (18), while there were 3 longitudinal cohort studies and 1 randomized controlled trial (RCT). The number of participants enrolled in the observational studies ranged from 100 to 7857. Most studies (19) were conducted on adults, while one ${ }^{20}$ included participants over 15 years of age, one sample ${ }^{21}$ was based on caregiver-child dyads, and one study was conducted on adolescents $13-15$ years old. ${ }^{22}$

The studies included in our review were conducted in various parts of the world, including Europe (Denmark-1, Finland-1, The Netherlands - 2, and United Kingdom-2), America (Canada-1 and United States-5), Australia-3, and Asia (China-1, Iran-1, Japan-3, and Taiwan-1). HL was most often measured with the Test of Functional Health Literacy in Adults ${ }^{23}$ or its shortened version, ${ }^{24}$ which was used in 7 studies. ${ }^{21,25-30}$ Other HL measures used are listed in Table 1 . The studies considered here used different tools to measure HL, making the results difficult to compare. The use of standardized and validated measuring instruments in future studies would facilitate comparisons between international findings.

The studies varied in their approach to measuring PA and mostly relied on self-reported information. In the majority of cases, participants answered questionnaires about the amount of time they usually spent on physical exercise (eg, weekly frequency) and its level of intensity (eg, mild, moderate, or vigorous).

Table 1 summarizes the methodology of the observational studies identified and included in the review. Table 2 shows the results of the studies.

Supplementary Material 2 (available online) illustrates the methodological quality of the studies. The 21 observational studies' STROBE scores are reported in Supplementary Material 2, attached 1 and 2 (available online). The single intervention study (an RCT) complied with $88.2 \%$ of the items of the CONSORT checklist (Supplementary Material 2, attached 3 [available online]).

Of the 21 observational studies, 17 found a positive association between HL and PA, providing evidence of higher levels of PA among individuals with higher levels of HL.

\section{Association Between HL and PA in Children}

Among all the studies analyzed, only one focused on parent-child dyads. This study ${ }^{21}$ enrolled parents of 2-month-old children, aiming to examine the association between the parents' HL and their infant care behavior that might be considered "obesogenic," such as feeding content, feeding style, and PA-related behavior. An inadequate amount of time spent in the prone position while awake ("tummy time" for less than $30 \mathrm{~min} / \mathrm{d}$ ) and an excessive amount of time spent watching television were considered obesogenic behaviors that limited the infant's PA. The results showed that parents with a low HL had nearly twice the odds of reporting that their infant spent time in front of the television, and 3 times higher odds of reporting an inadequate daily "tummy time."

\section{Association Between HL and PA in Adolescents}

One of the studies focused on school-aged adolescents (13 or $15 \mathrm{y}$ old), aiming to compare the levels of HL among those who did or 


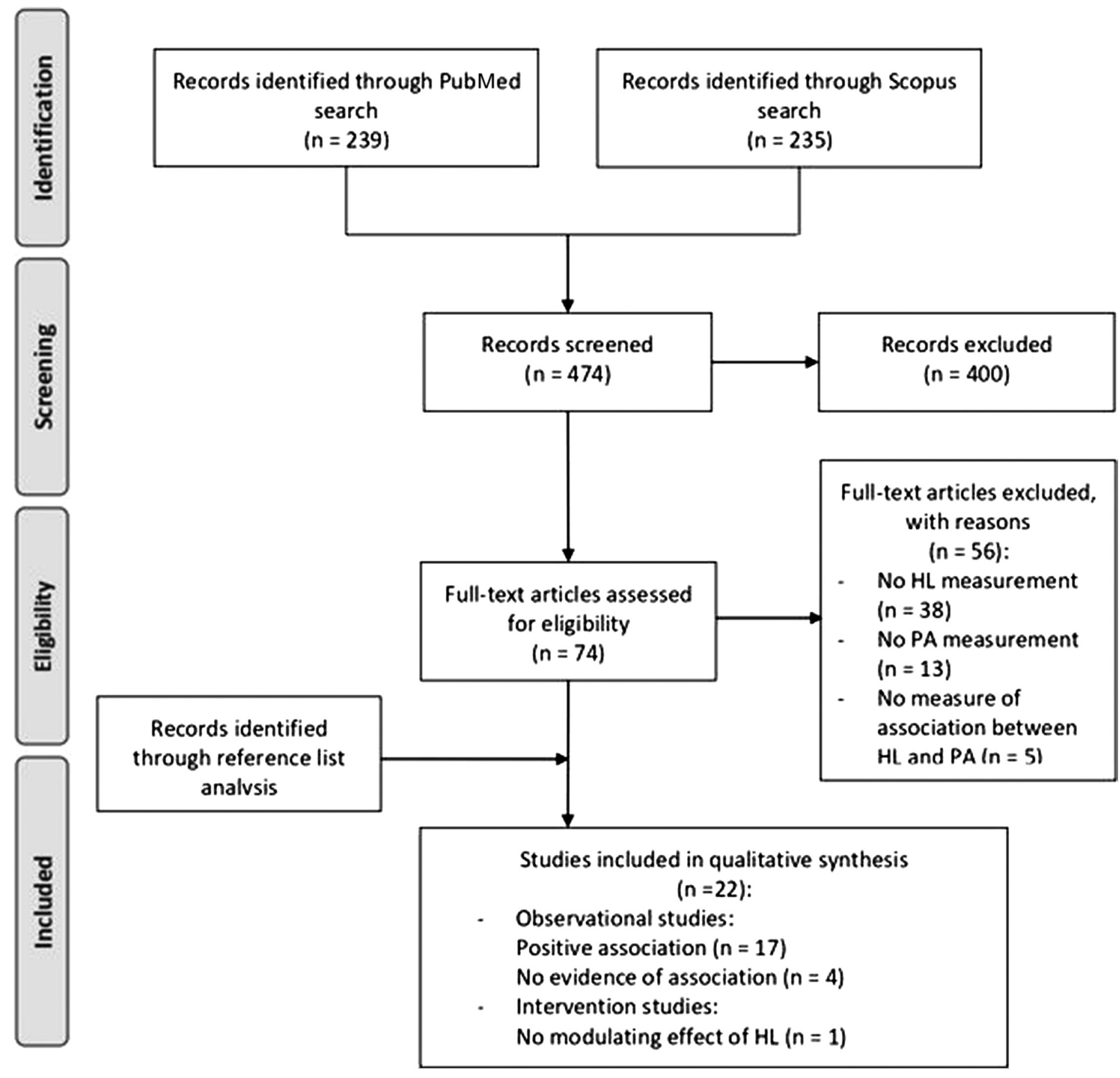

Figure 1 - Flow diagram of article selection process. HL indicates health literacy; PA, physical activity.

did not participate in sports club activities. ${ }^{22}$ The findings indicated that adolescents who were members of sports clubs had a higher HL than nonmembers, regardless of age or gender. ${ }^{22}$

\section{Association Between HL and PA in Adults and Elderly}

Among the other 19 observational studies focusing on adults or older adults, 15 demonstrated a positive association between PA and HL. One ${ }^{35}$ was based on 18- to 24-year-old college students, and aimed to examine whether individual factors (health status, degree of health concern, major, and frequency of discussion about health-related issues in particular) and eHealth literacy affected their health-related behavior, for example, eating, PA, and sleeping. The study found that eHealth literacy mediated the association between individual factors and health-related behavior: higher levels of critical eHealth literacy prompted students to adopt multiple behaviors positive for their health, including PA. Another study $^{31}$ investigated whether HL mediates the association between educational attainment and health-related behavior, including PA. The study sample included adults aged 25 years or older participating in a large population-based survey $(\mathrm{N}=29,473)$. The results 


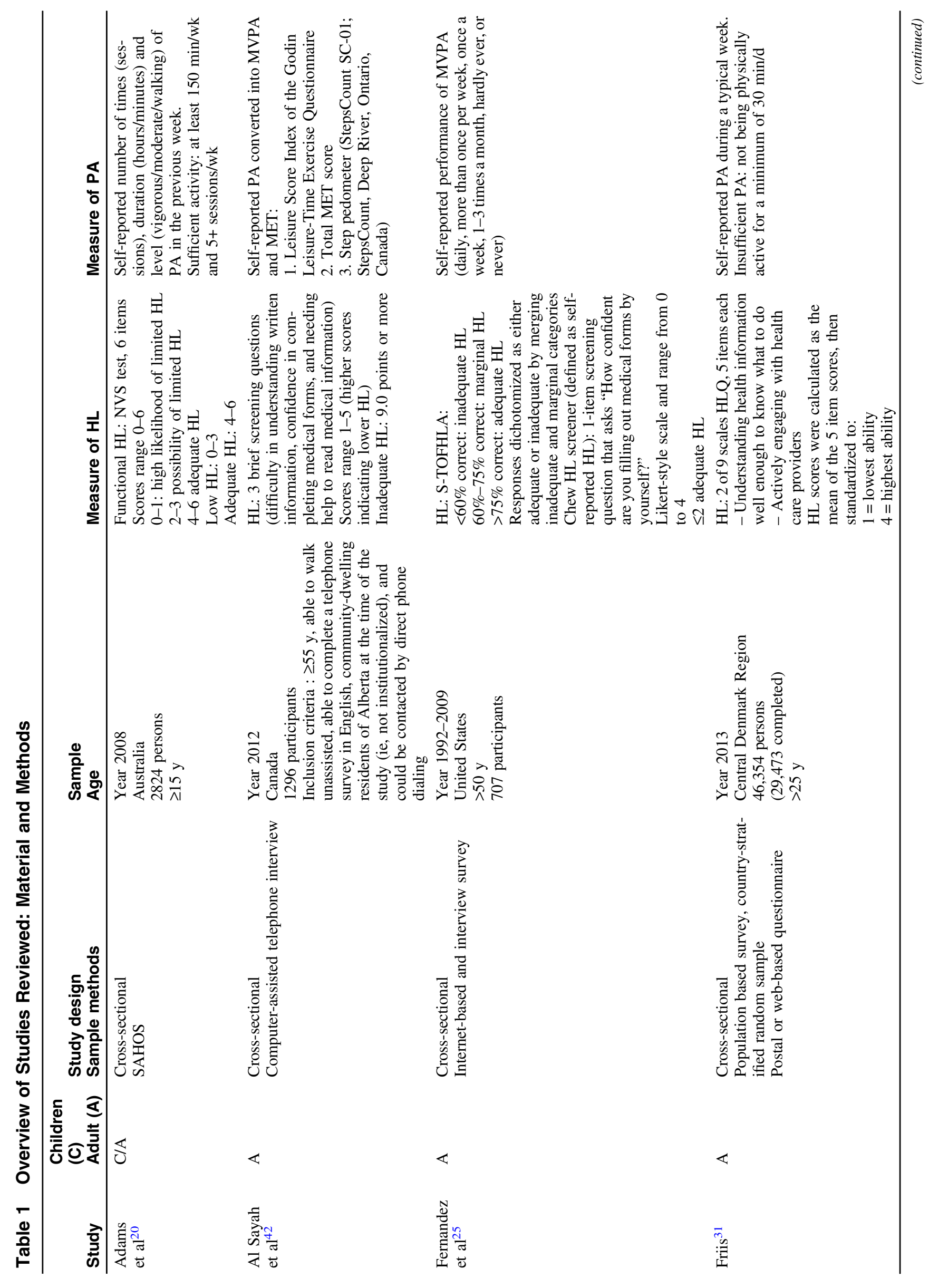




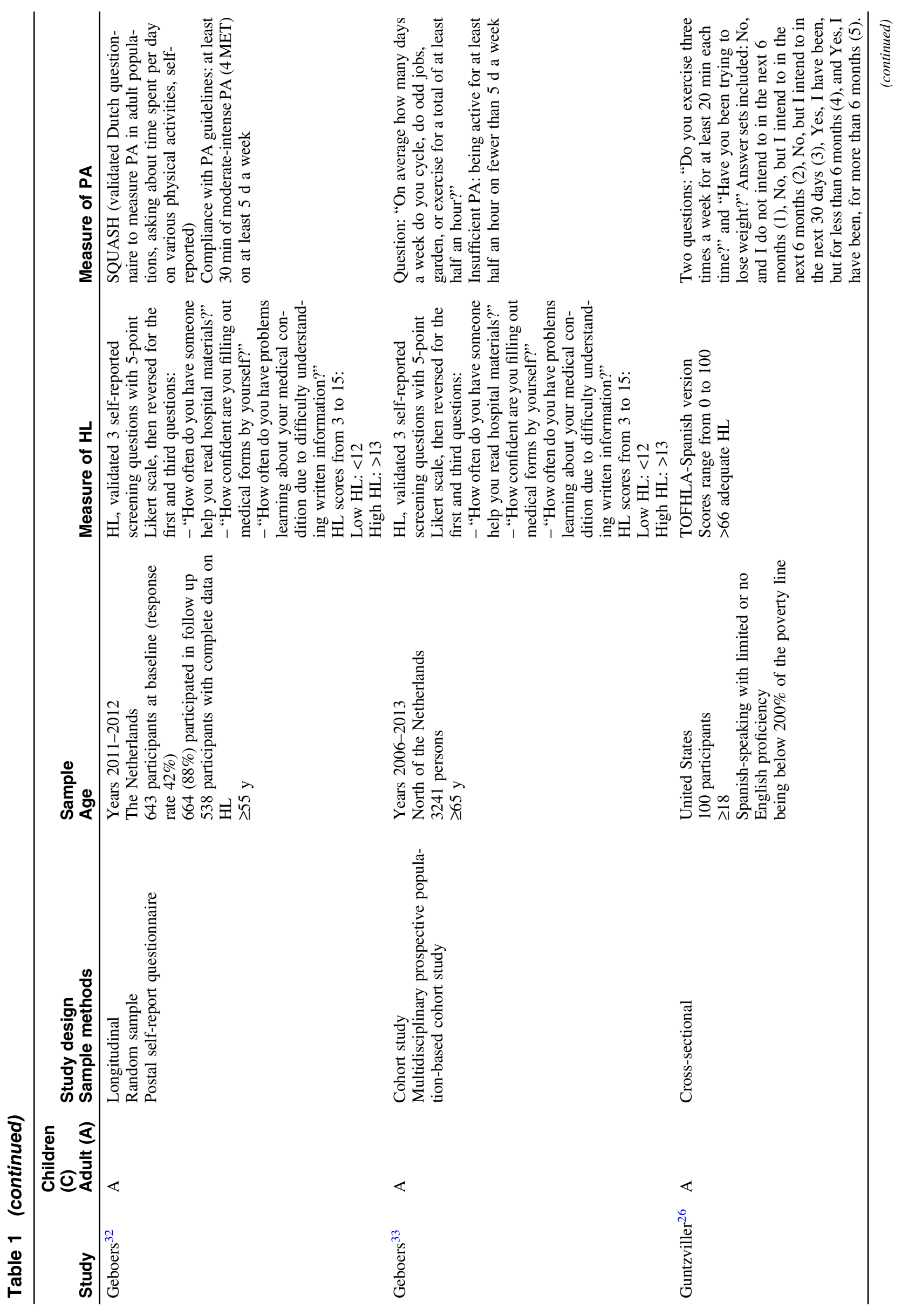




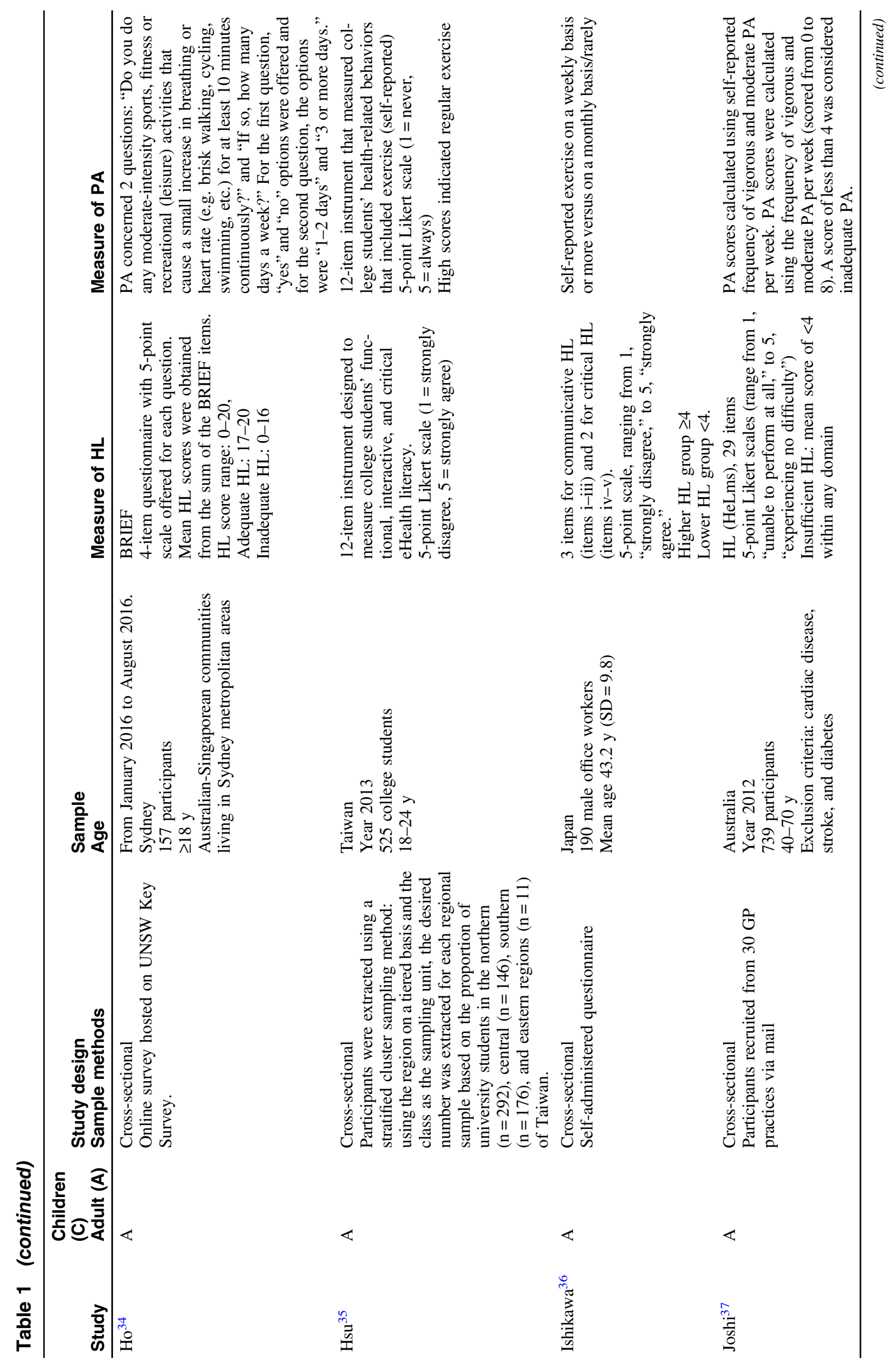




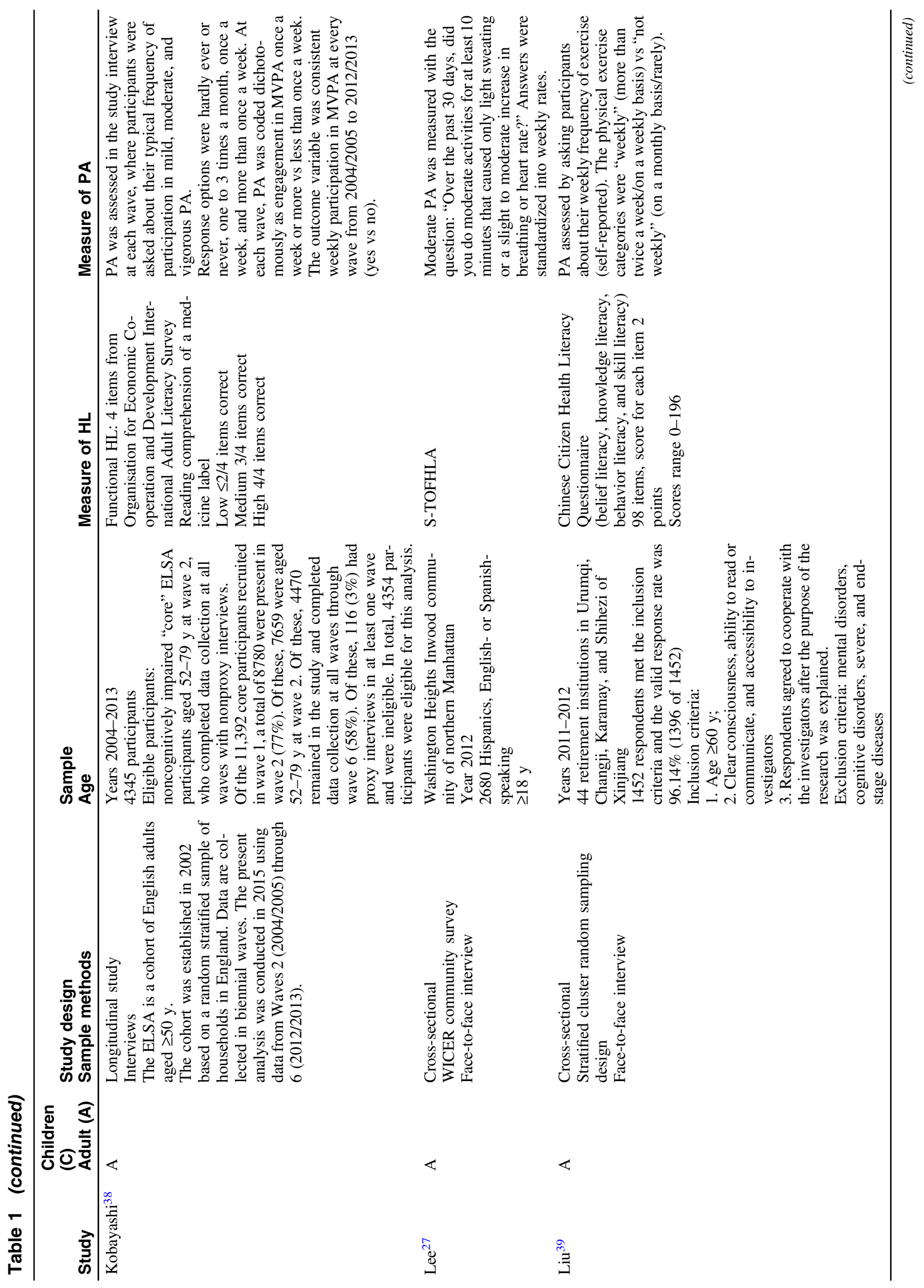




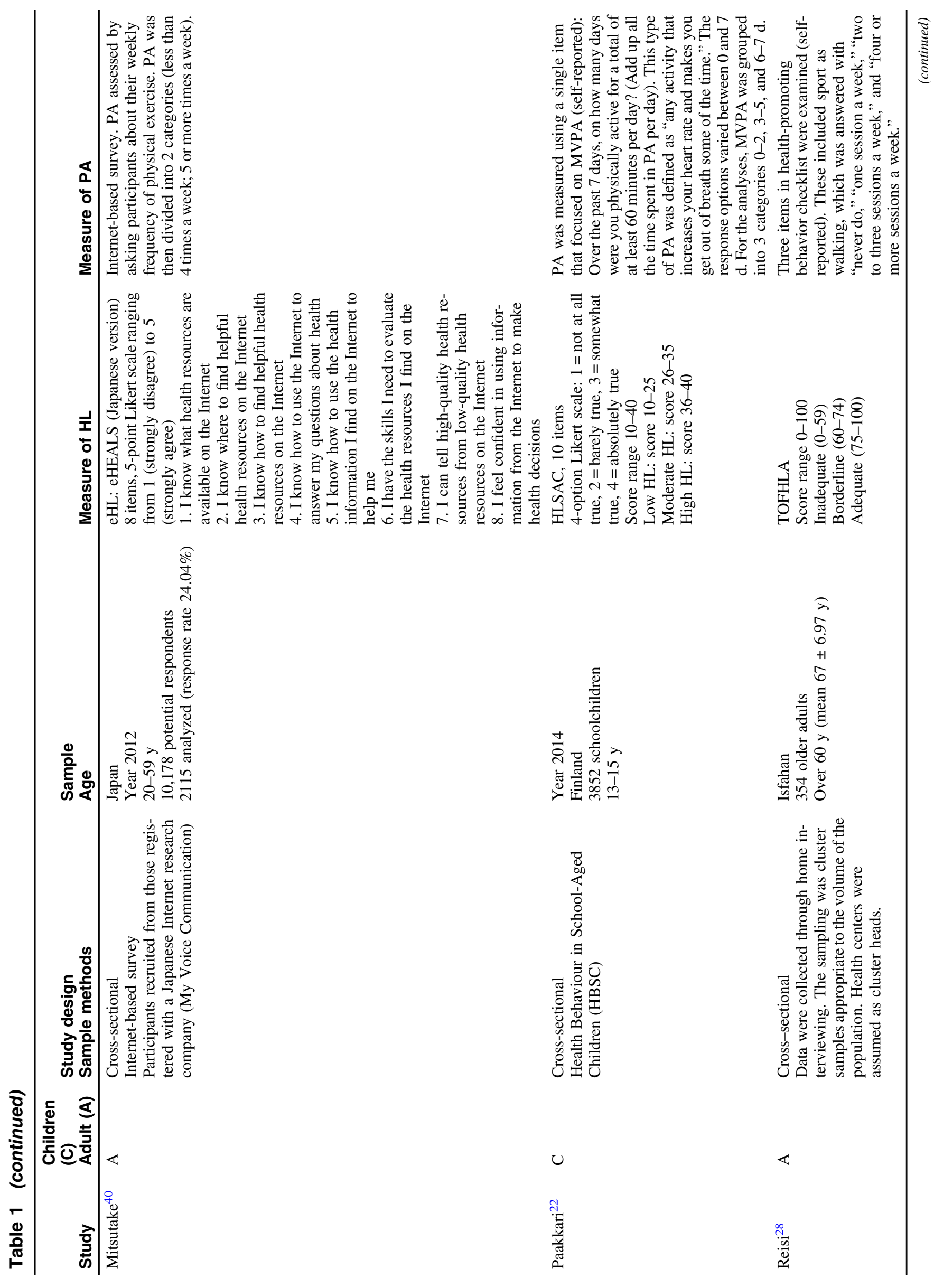




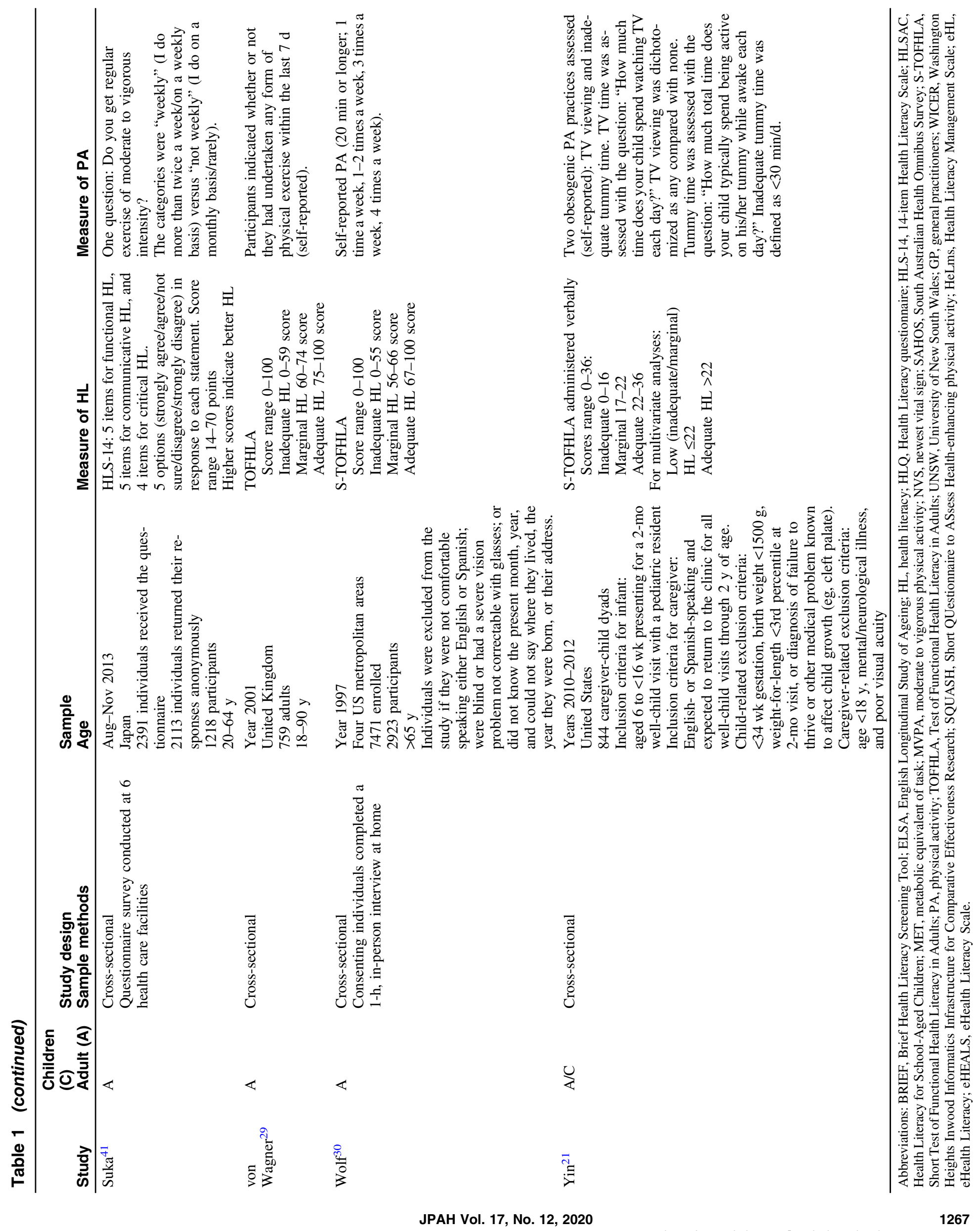




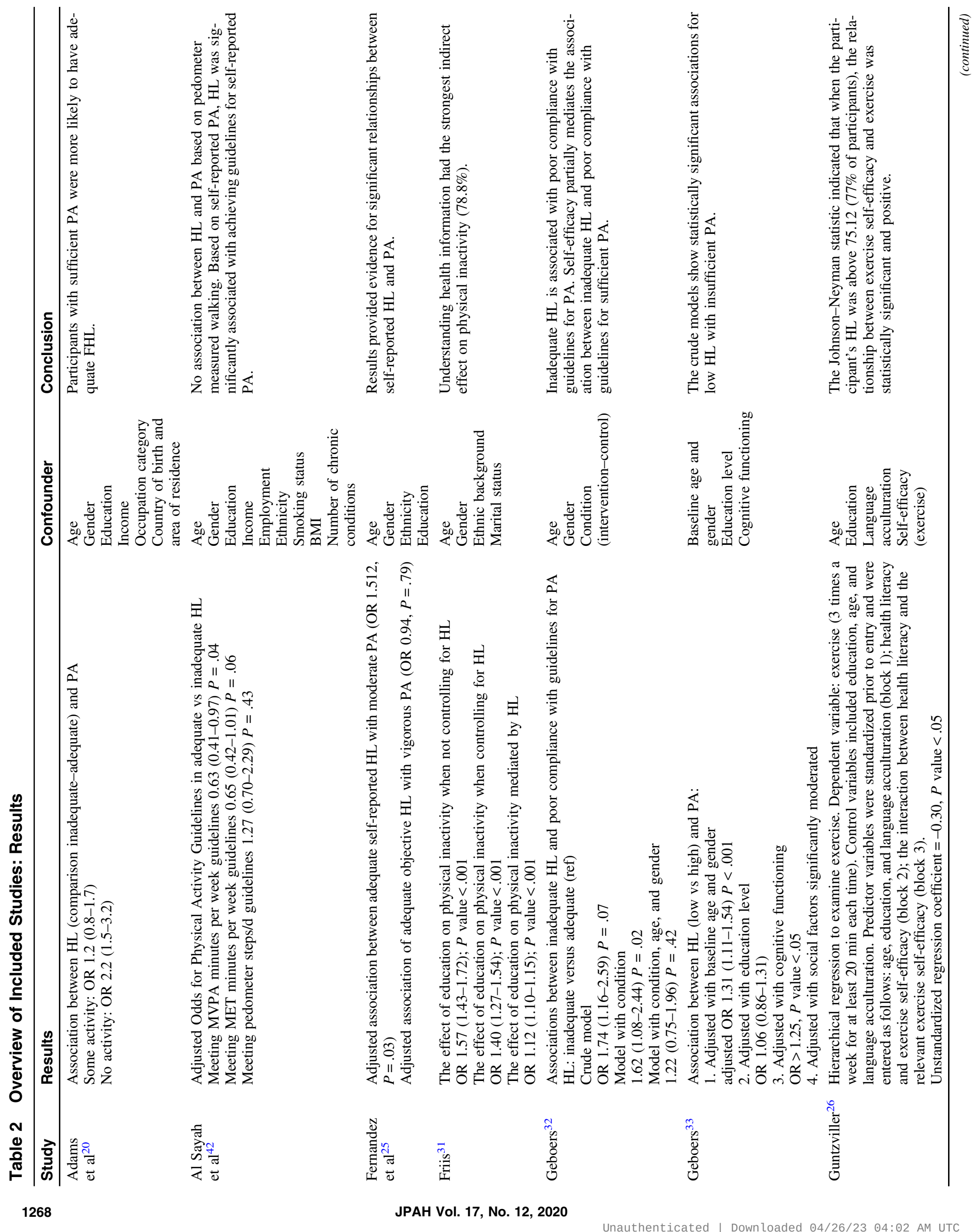



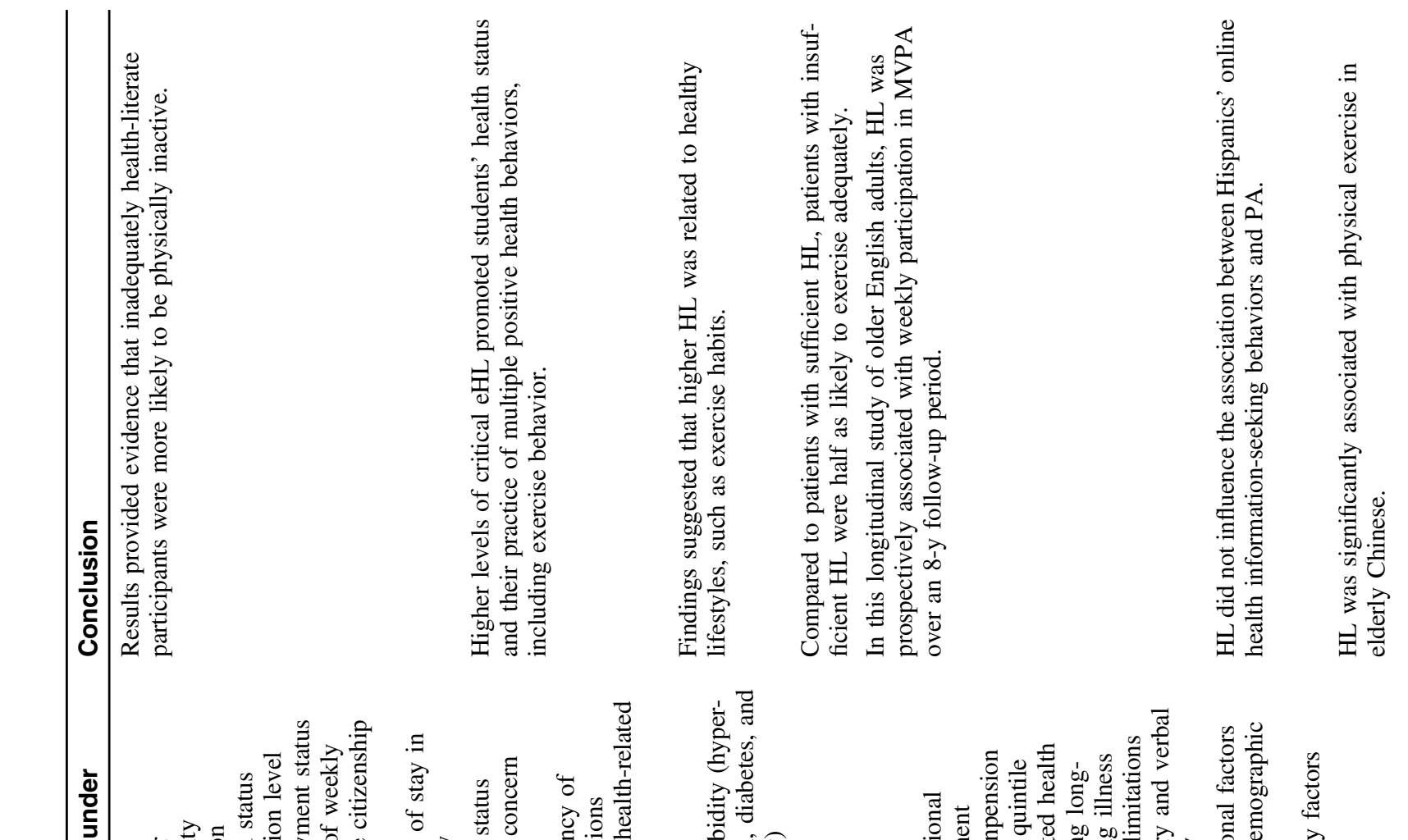

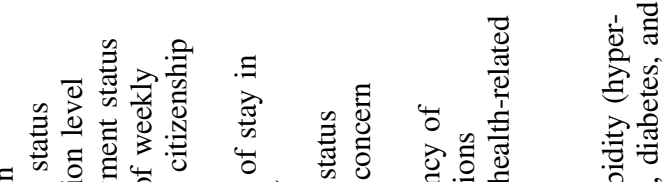

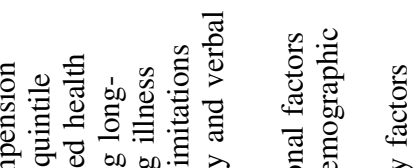
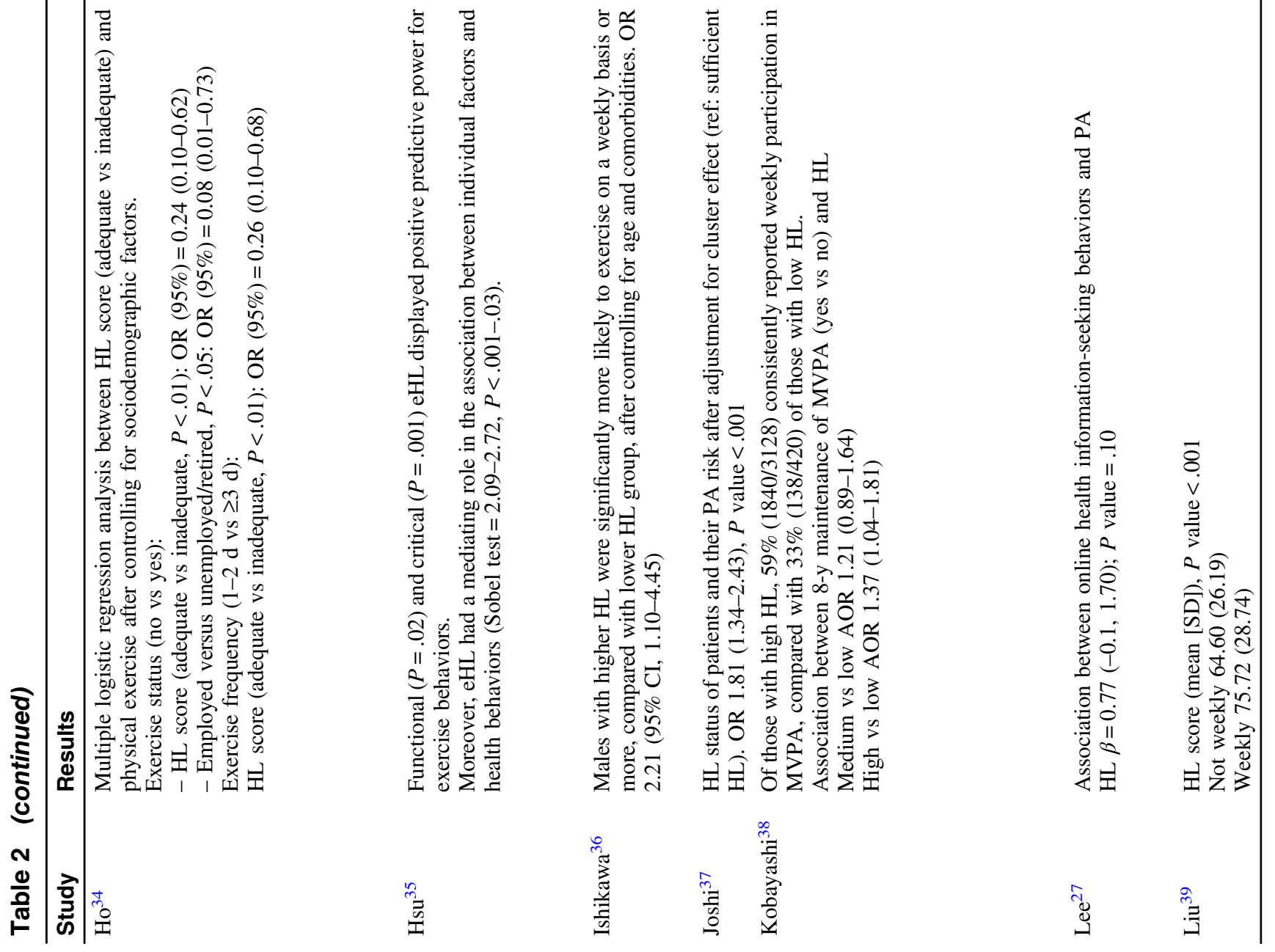


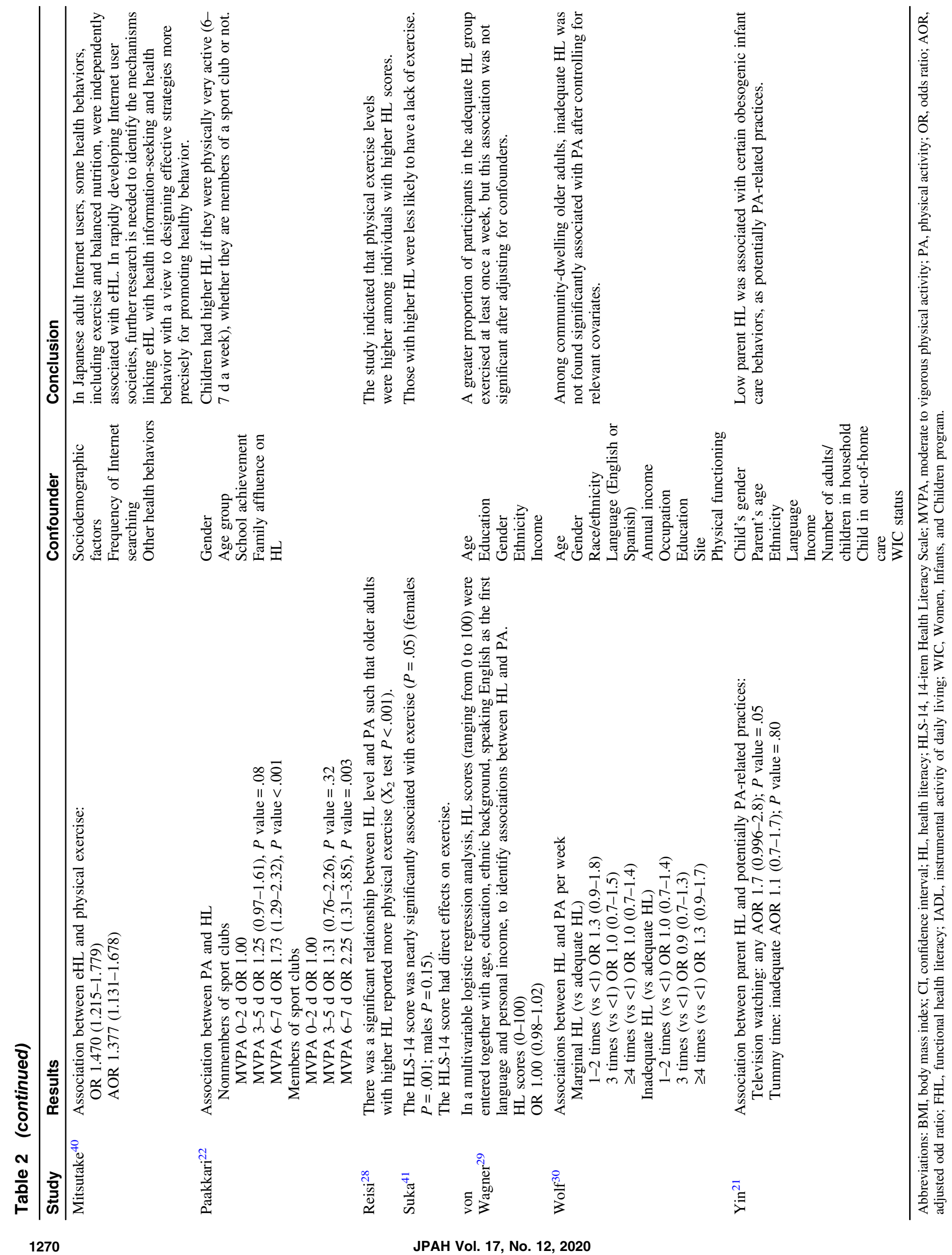


showed that HL in general, and the ability to understand health information in particular, mediated the relationship between educational attainment and health-related behavior, especially as regards being physically active for at least 30 minutes a day.

Eight of the studies concerned older adults, and 6 of them showed a positive association between $\mathrm{HL}$ and PA. One, in particular, ${ }^{25}$ specifically differentiated moderate PA from vigorous PA, and found HL positively associated only with moderate PA (performed 2 or more times a week), whereas there was no association between the 2 variables when vigorous PA was considered.

Four publications reported no statistically significant association between HL and PA. They were all cross-sectional studies and focused on adult populations. For example, Al Sayah et $\mathrm{al}^{42}$ found no association between HL and PA based on walking measured with a pedometer, although HL was significantly associated with compliance with the guidelines for self-reported PA. In order to clarify this discrepancy in the results, further studies are needed on the association between HL and an objective measure of PA.

The use of a meta-analysis for observational studies was prevented by the different methods used to assess HL in the various studies included in the review. This heterogeneity makes it impossible to combine the results of different studies, as it would lead to a methodological error.

Only one intervention study ${ }^{43}$ emerged from our literature search, which examined the modifying effect of HL on interventions designed to promote PA. The study examined adherence to a culturally and individually tailored Internet-based PA intervention for Spanish-speaking Latinas. This RCT was conducted in the United States, enrolling and randomizing a sample of 205 women aged 18-65 years, with a body mass index $<45 \mathrm{~kg} / \mathrm{m}^{2}$, who identified themselves as Hispanic or Latina, were able to read Spanish fluently, and self-reported insufficient PA (defined as less than 60 min a week of moderate to vigorous PA). HL was measured with the Spanish Short Test of Functional Health Literacy in Adults. Participants were offered an online intervention that included a culturally and individually tailored PA program, an opportunity for self-monitoring, goal setting, the possibility to anonymously ask health-related questions to a PhD-level researcher, social support among participants, online resources such as maps to prepare walking routes, and free exercise videos. A control group was given access to a website that offered information on health-related topics other than PA. The study examined the differences between the intervention and control groups in terms of minutes per week of moderate to vigorous physical activity (MVPA). The intervention group reported significantly more MVPA at 12 months than the control group. The authors also analyzed several baseline variables that might moderate the efficacy of the intervention, and HL did not emerge as a significant moderator.

\section{Discussion}

This systematic review showed that most of the observational studies considered (17 out of 21) consistently found a positive association between PA and HL: individuals with a more active lifestyle tended to have higher levels of HL than those who were more sedentary, suggesting that an adequate $\mathrm{HL}$ is a reliable correlate of regular PA. This positive association between HL and PA also emerged consistently in different age groups. In particular, the only study included in this review that involved parent-child dyads ${ }^{21}$ found parents of 2-month-old infants with a low HL associated with certain infant care behaviors that are considered "obesogenic": they were nearly twice as likely to report that their infant watched television and 3 times as likely to report an inadequate daily "tummy time." This would mean that a parent's low HL could influence their child's attitude to PA-related practices as they grow up.

Though the studies under review adopted different measures of both HL and PA, the results seemed quite consistent: the higher the HL, the more the PA. To be more specific, the articles considered several different performance-based (ie, objective) or self-reported (ie, subjective) measures of HL that focused on different constructs. The use of such diverse tools might be expected to generate dissimilar results, ${ }^{44,45}$ but the association between HL and PA seemed to be independent of the HL construct being measured, unlike the situation seen for disease prevention behavior, such as vaccination acceptance. ${ }^{46}$

Overall, the positive association between HL and PA found in the majority of the studies reviewed can be explained by the fact that individuals with a better-developed HL have skills and capabilities that enable them to engage in various forms of personal healthenhancing behavior, ${ }^{7}$ such as regular PA. HL is not only about an individual's ability to obtain, process, and understand basic health information and services, and to make appropriate health-related decisions. ${ }^{10}$ It also induces individuals to exert a greater control over their health and health-related decisions, with a practical fallout on everyday life. ${ }^{47}$ This notion is specifically conceptualized by the 2 higher levels of HL identified by Nutbeam ${ }^{47}$-interactive HL and critical HL. Interactive HL refers to the development of personal skills that enable individuals to improve their own ability, motivation and self-confidence to act on advice received. Critical HL reflects the cognitive and skill development outcomes oriented toward supporting effective social and political action, as well as individual action. ${ }^{47}$ Individuals with an adequate interactive and critical HL are capable of changing their personal lifestyle and adopting healthy habits. ${ }^{3,47}$

As regards the possible moderating effect of HL on the outcomes of health promotion interventions designed to improve the population's adherence to an active lifestyle, the one experimental study ${ }^{43}$ reviewed was a RCT comparing intervention and control groups in terms of minutes per week of MVPA. At 12 months, the intervention group reported significantly more MVPA than the control group. HL did not significantly moderate the efficacy of the intervention, however, whereas BMI had a significant modifying effect on self-reported MVPA. This lack of any moderating effect of HL suggests that the intervention was effective regardless of participants' level of HL. In other words, such health promotion interventions can prompt behavioral changes, making individuals more physically active, whatever their HL. This means that health promotion interventions to improve the population's knowledge of health-related topics could overcome inequalities in the adoption of healthy lifestyles caused by differences in education and socioeconomic status. In particular, an inadequate HL could negatively influence the personal motivation, ${ }^{48}$ problem-solving ability, ${ }^{49}$ self-efficacy, ${ }^{50}$ and/or awareness $^{51}$ needed to implement proper self-care behavior patterns. Interventions based on an educational approach could thus mitigate the influence of HL at baseline and make individuals aware of the importance of their lifestyle to their health outcomes.

Overall, this study confirmed that HL is an important predictor of an active lifestyle, consistently with other studies finding that $\mathrm{HL}$ is one of the main predictors of the effectiveness of health promotion and disease prevention programs. ${ }^{3}$ This means that public health would benefit from nurturing HL in the general population. Since only one RCT was available for our review, 
further experimental studies are needed to support this evidence. HL can be seen as the result of health education strategies that enable the population to take more responsibility for their daily health-related decisions and behavior, ${ }^{4}$ so improving HL is a goal of individual medicine and public health. If future research confirms these results, governments should incorporate the notion and paradigm of HL in their policy-making, research programs, and population health goals. A "health-literate society" can only be achieved by taking a multidisciplinary and comprehensive approach, targeting health services, the education sector, the workplace, and community organizations.

\section{Limitations}

Our systematic review has several limitations. First, the studies considered here used different tools to measure both HL and PA, making the results difficult to compare and preventing any metaanalysis. The use of standardized and validated measuring instruments in future studies would facilitate comparisons between international findings. Second, many of the samples considered in our review were drawn from particular categories of the population that might be seen as vulnerable (ie, older adults or ethnic minorities) and may not be representative of the general population. The characteristics of the target population could influence the level of association between HL and PA, and further studies should try to clarify the role of different sociodemographic characteristics possibly mediating HL and health-related behavior, such as the influence of age on the association between the 2 variables. We also found only one publication concerning an intervention program that enabled us to examine whether HL mediated the efficacy of the intervention. This study was performed in women with a specific ethnic and cultural background, so more comprehensive studies are needed to shed further light on these topics.

\section{Conclusions}

Most of the literature examined found that a higher HL was associated with higher levels of PA. This means that it is important to improve individuals' HL so that they can make better-informed decisions about their lifestyle and levels of PA. By doing so, they can avoid diseases and disabilities associated with a sedentary lifestyle, reducing the prevalence of many noncommunicable chronic conditions.

In the only intervention study available, HL did not emerge as a significant moderator of the outcome of interventions to improve levels of PA, suggesting that such programs have the potential to help reduce health inequalities in the population at large. More studies will be needed to support this tentative conclusion, however.

\section{Acknowledgments}

This research received no specific grant from any funding agency in the public, commercial, or not-for-profit sectors. The authors declare that there is no conflict of interest.

\section{References}

1. WHO. Global Action Plan for the Prevention and Control of NCDs 2013-2020. Geneva, Switzerland: WHO Press, World Health Organization; 2013.
2. WHO. Shanghai declaration on promoting health in the 2030. Agenda for Sustainable Development. Health Promot Int. 2017;32(1):7.

3. Nutbeam D. Health literacy as a population strategy for health promotion. JJHEP. 2017;25(3):210-222. doi:10.11260/kenkokyoiku. 25.210

4. Kickbusch I, Maag D. Health literacy. In: International Encyclopedia of Public Health. Vol 3. 1st ed. San Diego, CA: Academic Press; 2008: 204-211.

5. Davis TC, Williams MV, Marin E, Parker RM, Glass J. Health literacy and cancer communication. CA Cancer J Clin. 2002;52(3): 134-149. PubMed ID: 12018928 doi:10.3322/canjclin.52.3.134

6. Schwartzberg JG, Claire Wang JV. Understanding Health Literacy: Implications for Medicine and Public Health. Chicago, IL: American Medical Association; 2005.

7. Nutbeam D. The evolving concept of health literacy. Soc Sci Med. 2008;67(12):2072-2078. PubMed ID: 18952344 doi:10.1016/j. socscimed.2008.09.050

8. Miller DP, Brownlee CD, McCoy TP, Pignone MP. The effect of health literacy on knowledge and receipt of colorectal cancer screening: a survey study. BMC Fam Pract. 2007;8(1):16. doi:10.1186/ 1471-2296-8-16

9. Pappas G, Siozopoulou V, Saplaoura K, Vasiliou A, Christou L, Akritidis N. Health literacy in the field of infectious diseases: the paradigm of brucellosis. J Infect. 2007;54(1):40-45. PubMed ID: 16533534 doi:10.1016/j.jinf.2006.01.018

10. Lynn Nielsen-Bohlman AMP, Kindig DA. Health Literacy: A Prescription to End Confusion. Washington, DC: The National Academies Press; 2004.

11. U.S. Department of Health and Human Services, Centers for Disease Control and Prevention. Improving health literacy for old adults. Expert panel report. 2009. http://www.cdc.gov/healthliteracy/Learn/ pdf/olderadults.pdf . Accessed 24 March 2020.

12. Cho YI, Lee SYD, Arozullah AM, Crittenden KS. Effects of health literacy on health status and health service utilization amongst the elderly. Soc Sci Med. 2008;66(8):1809-1816. PubMed ID: 18295949 doi:10.1016/j.socscimed.2008.01.003

13. Hills AP, Street SJ, Byrne NM. Physical activity and health. Adv Food Nutr Res. 2015;75:77-95. PubMed ID: 26319905 doi:10.1016/bs. afnr.2015.06.001

14. Fletcher GF, Landolfo C, Niebauer J, Ozemek C, Arena R, Lavie CJ. Promoting physical activity and exercise: JACC health promotion series. J Am Coll Cardiol. 2018;72(23, pt B):3053-3070. PubMed ID: 30522636 doi:10.1016/j.jacc.2018.10.025

15. Lee IM, Shiroma EJ, Lobelo F, Puska P, Blair SN, Katzmarzyk PT; Lancet Physical Activity Series Working Group. Effect of physical inactivity on major non-communicable diseases worldwide: an analysis of burden of disease and life expectancy. Lancet. 2012; 380(9838):219-229. PubMed ID: 22818936 doi:10.1016/S01406736(12)61031-9

16. Powell KE, King AC, Buchner DM, et al. The Scientific Foundation for the Physical Activity Guidelines for Americans, 2nd Edition. J Phys Act Health. 2018;17:1-11. doi:10.1123/jpah.2018-0618

17. von Elm E, Altman DG, Egger M, Pocock SJ, Gøtzsche PC, Vandenbroucke JP; STROBE Initiative. The Strengthening the Reporting of Observational Studies in Epidemiology (STROBE) statement: guidelines for reporting observational studies. J Clin Epidemiol. 2008;61(4):344-349. PubMed ID: 18313558 doi:10. 1016/j.jclinepi.2007.11.008

18. Schulz KF, Altman DG, Moher D; CONSORT Group. CONSORT 2010 statement: updated guidelines for reporting parallel group randomized trials. Ann Intern Med. 2010;152(11):726-732. doi:10. 7326/0003-4819-152-11-201006010-00232 
19. Altman D. (1991). Practical Statistics for Medical Research. London, UK: Chapman and Hall.

20. Adams RJ, Piantadosi C, Ettridge K, et al. Functional health literacy mediates the relationship between socio-economic status, perceptions and lifestyle behaviors related to cancer risk in an Australian population. Patient Educ Couns. 2013;91(2):206-212. PubMed ID: 23276386 doi:10.1016/j.pec.2012.12.001

21. Yin HS, Sanders LM, Rothman RL, et al. Parent health literacy and "obesogenic" feeding and physical activity-related infant care behaviors. J Pediatr. 2014;164(3):577-583.e1. PubMed ID: 24370343 doi:10.1016/j.jpeds.2013.11.014

22. Paakkari L, Kokko S, Villberg J, Paakkari O, Tynjälä J. Health literacy and participation in sports club activities among adolescents. Scand J Public Health. 2017;45(8):854-860. PubMed ID: 28673131 doi:10.1177/1403494817714189

23. Nurss JR, Parker RM, Baker DW. TOFHLA: Test of Functional Health Literacy in Adults. 2nd ed. Snow Camp, NC: Peppercorn Books and Press; 2001.

24. Baker DW, Williams MV, Parker RM, Gazmararian JA, Nurss J. Development of a brief test to measure functional health literacy. Patient Educ Couns. 1999;38(1):33-42. PubMed ID: 14528569 doi:10.1016/S0738-3991(98)00116-5

25. Fernandez DM, Larson JL, Zikmund-Fisher BJ. Associations between health literacy and preventive health behaviors among older adults: findings from the Health and Retirement Study. BMC Public Health. 2016;16(1):596. PubMed ID: 27430477 doi:10.1186/s12889016-3267-7

26. Guntzviller LM, King AJ, Jensen JD, Davis LA. Self-efficacy, health literacy, and nutrition and exercise behaviors in a low-income, Hispanic population. J Immigr Minor Health. 2017;19(2):489-493. PubMed ID: 26979167 doi:10.1007/s10903-016-0384-4

27. Lee YJ, Boden-Albala B, Jia H, Wilcox A, Bakken S. The association between online health information-seeking behaviors and health behaviors among Hispanics in New York City: a community-based cross-sectional study. J Med Internet Res. 2015;17(11):e261. PubMed ID: 26611438 doi:10.2196/jmir.4368

28. Reisi M, Javadzade SH, Heydarabadi AB, Mostafavi F, Tavassoli $\mathrm{E}$, Sharifirad G. The relationship between functional health literacy and health promoting behaviors among older adults. J Educ Health Promot. 2014;3(1):119. doi:10.4103/2277-9531.145925

29. von Wagner C, Knight K, Steptoe A, Wardle J. Functional health literacy and health-promoting behaviour in a national sample of British adults. J Epidemiol Community Health. 2007;61(12):10861090. PubMed ID: 18000132 doi:10.1136/jech.2006.053967

30. Wolf MS, Gazmararian JA, Baker DW. Health literacy and health risk behaviors among older adults. Am J Prev Med. 2007;32(1):19-24. PubMed ID: 17184964 doi:10.1016/j.amepre.2006.08.024

31. Friis K, Lasgaard M, Rowlands G, Osborne RH, Maindal HT. Health literacy mediates the relationship between educational attainment and health behavior: a Danish population-based study. J Health Commun. 2016;21(suppl 2):54-60. PubMed ID: 27668691 doi:10.1080/ 10810730.2016.1201175

32. Geboers B, de Winter AF, Luten KA, Jansen CJ, Reijneveld SA. The association of health literacy with physical activity and nutritional behavior in older adults, and its social cognitive mediators. $J$ Health Commun. 2014;19(suppl 2):61-76. doi:10.1080/10810730.2014. 934933

33. Geboers B, Reijneveld SA, Jansen CJ, de Winter AF. Health literacy is associated with health behaviors and social factors among older adults: results from the LifeLines Cohort Study. J Health Commun. 2016;21(suppl 2):45-53. PubMed ID: 27661472 doi:10.1080/ 10810730.2016.1201174

34. Ho TG, Hosseinzadeh H, Rahman B, Sheikh M. Health literacy and health-promoting behaviours among Australian-Singaporean communities living in Sydney metropolitan area. Proc Singapore Healthc. 2018;27(2):125-131. doi:10.1177/2010105817741906

35. Hsu W, Chiang C, Yang S. The effect of individual factors on health behaviors among college students: the mediating effects of eHealth literacy. J Med Internet Res. 2014;16(12):e287. PubMed ID: 25499086 doi:10.2196/jmir.3542

36. Ishikawa H, Nomura K, Sato M, Yano E. Developing a measure of communicative and critical health literacy: a pilot study of Japanese office workers. Health Promot Int. 2008;23(3):269-274. PubMed ID: 18515303 doi:10.1093/heapro/dan017

37. Joshi C, Jayasinghe UW, Parker S, et al. Does health literacy affect patients' receipt of preventative primary care? A multilevel analysis. BMC Fam Pract. 2014;15(1):171. doi:10.1186/s12875-014-0171-z

38. Kobayashi LC, Wardle J, Wolf MS, von Wagner C. Health literacy and moderate to vigorous physical activity during aging, 2004-2013. Am J Prev Med. 2016;51(4):463-472. PubMed ID: 27140501 doi:10. 1016/j.amepre.2016.02.034

39. Liu YB, Liu L, Li YF, Chen YL. Relationship between health literacy, health-related behaviors and health status: a survey of elderly Chinese. Int J Environ Res Public Health. 2015;12(8):9714-9725. PubMed ID: 26295246 doi:10.3390/ijerph120809714

40. Mitsutake S, Shibata A, Ishii K, Oka K. Associations of eHealth literacy with health behavior among adult internet users. $J$ Med Internet Res. 2016;18(7):e192. PubMed ID: 27432783 doi:10.2196/ jmir.5413

41. Suka M, Odajima T, Okamoto M, et al. Relationship between health literacy, health information access, health behavior, and health status in Japanese people. Patient Educ Couns. 2015;98(5):660-668. PubMed ID: 25739344 doi:10.1016/j.pec.2015.02.013

42. Al Sayah F, Johnson ST, Vallance J. Health literacy, pedometer, and self-reported walking among older adults. Am J Public Health. 2016;106(2):327-333. PubMed ID: 26691129 doi:10.2105/AJPH. 2015.302901

43. Hartman SJ, Dunsiger SI, Bock BC et al. Physical activity maintenance among Spanish-speaking Latinas in a randomized controlled trial of an Internet-based intervention. J Behav Med. 2017; 40(3):392-402. PubMed ID: 27752866 doi:10.1007/s10865-0169800-4

44. Kiechle ES, Bailey SC, Hedlund LA, Viera AJ, Sheridan SL. Different measures, different outcomes? A systematic review of performance-based versus self-reported measures of health literacy and numeracy. J Gen Intern Med. 2015;30(10):1538-1546. PubMed ID: 25917656 doi:10.1007/s11606-015-3288-4

45. Nguyen TH, Paasche-Orlow MK, McCormack LA. The state of the science of health literacy measurement. Inf Serv Use. 2017;37(2): 189-203. doi:10.3233/ISU-170827.

46. Lorini C, Santomauro F, Donzellini M, et al. Health literacy and vaccination: a systematic review. Hum Vaccin Immunother. 2018; 14(2):478-488. doi:10.1080/21645515.2017.1392423

47. Nutbeam D. Health literacy as a public health goal: a challenge for contemporary health education and communication strategies into the 21st century. Health Promot Int. 2000;15(3):259-267. doi:10.1093/ heapro/15.3.259

48. Verhoeven L, Snow C, eds. Literacy and Motivation: Reading Engagement in Individuals and Groups. Mahwah, NJ: Lawrence Erlbaum Associates Publishers; 2001: 326 p.vii. 
49. Kaminski RA, Good RH III, eds. Assessing Early Literacy Skills in a Problem-Solving Model: Dynamic Indicators of Basic Early Literacy Skills. Eugene, OR: Mark R. Shinn; 1998.

50. Torres RY, Marks R. Relationships among health literacy, knowledge about hormone therapy, self-efficacy, and decision-making among postmenopausal health. J Health Commun. 2009;14(1):
43-55. PubMed ID: 19180370 doi:10.1080/10810730802 592247

51. Wolf MS, Davis TC, Cross JT, Marin E, Green K, Bennett CL. Health literacy and patient knowledge in a Southern US HIV clinic. Int J STD AIDS. 2004;15(11):747-752. PubMed ID: 15537462 doi:10.1258/ 0956462042395131 